\title{
UNIVERSIDAD SANTO TOMÁS Y GLOBALIZACIÓN: INICIATIVAS PARA AFRONTAR ESTE PROCESO
}

Con el fin de incrementar iniciativas concretas entre las universidades socias del Consejo Internacional de Universidades Santo Tomás de Aquino (IC-USTA), que apoyen la equidad entre docentes y estudiantes de diversos lugares del mundo en el acceso a la información y el conocimiento, la Universidad Santo Tomás (UST) de Chile ha desarrollado diversas iniciativas que van más allá del intercambio de estudiantes y profesores, y que abarcan los ámbitos de docencia, investigación y extensión. Entre éstas destaca la Cátedra Globalización, desarrollada por cinco universidades de este consorcio con el fin de dar respuesta al necesario intercambio cultural que debe existir hoy en la formación universitaria.

Además, en el ámbito de la investigación aplicada al mundo de los negocios se desarrollaron, a comienzos de la década, estándares de gobiernos corporativos en conjunto entre las universidades Santo Tomás de Santiago de Chile y de Houston. Esta investigación, que aprovechó la experiencia disponible en este ámbito en la red IC-USTA, puso a disposición de la comunidad de negocios un instrumento desarrollado sobre la base de estándares internacionales y cuya aplicación facilita el levantamiento de capital para las empresas y la toma de decisiones de inversión nacional e internacional.

En el área académica, en tanto, destacan iniciativas como la exportación de la metodología de enseñanza y aprendizaje Syllabus desde la UST de Chile a la de Houston, implementación de centros de aprendizajes para los alumnos, iniciativa que fue importada desde Houston y, en extensión, conferencias de académicos de las 
instituciones pertenecientes al consorcio, realización de congresos internacionales en conjunto y adopción de iniciativas de otras universidades, como es el caso del Congreso Católicos y Vida Pública.

Este artículo describe estas experiencias desarrolladas por la Universidad Santo Tomás de Chile y miembros de la red IC-USTA, aprovechando el contexto que otorga la globalización en el ámbito de la educación superior. Estas experiencias pueden ser de utilidad para académicos y estudiantes de la educación superior del país.

\section{Historia de IC-USTA}

Internacional Council of the Universities of Saint Thomas Aquinas (IC-USTA) es una red mundial compuesta por 23 universidades, con presencia en los cinco continentes, que comparten el espíritu y los principios intelectuales, pedagógicos y metodológicos de Santo Tomás de Aquinoi. El consorcio fue fundado desde la Universidad Santo Tomás de Chile en 1993 y, en la actualidad, reúne a más de 250 mil estudiantes, quienes pueden acceder a los programas de intercambio académico existentes, los cuales, además de ofrecer conocimientos técnicos y profesionales sobre las materias, entregan el pensamiento de Santo Tomás de Aquino aplicado a los problemas actuales.

La fundación de IC-USTA adquiere hoy mayor importancia debido al proceso de globalización. Existe la necesidad de relacionar las distintas culturas y experimentar la convivencia social, cultural y económica. Esta red de intercambio otorga la posibilidad de mejorar las competencias de nuestros alumnos en estos aspectos y ofrece la opción real de que experimenten el estudio y la interacción. Alrededor de medio centenar de estudiantes provenientes de las distintas sedes de la Universidad

i En el anexo se individualizan las universidades pertenecientes a esta red. 
Santo Tomás de Chile han realizado programas de intercambio con distintas universidades de la red ${ }^{\mathrm{ii}}$.

En términos de organización, el directorio de IC-USTA -formado por los rectores de las universidades socias- se reúne cada dos años en alguna de estas instituciones, donde se plantean los desafíos del consorcio para el próximo bienio.

\section{Cátedra Globalización}

Es una de las iniciativas conjuntas que han desarrollado cinco universidades de este consorcio, con el objetivo de dar respuesta al necesario intercambio cultural que debe existir actualmente en la formación universitaria.

El curso -que se realiza a distancia en español e inglés- se suma al programa de intercambios académicos de profesores y alumnos, y a los proyectos interdisciplinarios desarrollados por las instituciones de educación superior pertenecientes a esta red de conocimiento.

Con el fin de apoyar la equidad entre docentes y estudiantes de diversos lugares del mundo en el acceso a la información, en el curso participan profesores y alumnos de cinco universidades de América y Europa, quienes estudian el proceso de globalización.

ii Resulta interesante recordar las palabras del profesor de la University of Saint Thomas, Houston, y director ejecutivo de IC-USTA, Joseph McFadden, en su clase magistral del 27 de abril de 2006 en la Universidad Santo Tomás de Santiago, con ocasión de la inauguración del año académico. Señaló que la multiculturalidad está presente en todo el mundo y los líderes deben saber aprovecharla; el intercambio de ideas y experiencias es cada vez mayor, lo que debe verse como algo positivo. Reconoció que, desde hace años, utiliza la metodología de aprendizaje Syllabus para impartir su cátedra, y que permite al estudiante participar activamente en su proceso de aprendizaje, clave para la formación universitaria de hoy.

McFadden comentó los progresos que ha experimentado esta red desde sus inicios y planteó que los próximos desafíos están orientados al incremento del intercambio de alumnos y profesores; pero, sobre todo, al desarrollo de proyectos de investigación comunes, programas de justicia social, oferta de grados y cursos, y la exploración de cooperación tecnológica. 
En la tabla 1 se identifica a las instituciones de educación superior que han participado hasta este momento en el curso con su respectivo número de alumnos.

Esta iniciativa ha tenido una excelente acogida entre los alumnos de las universidades integrantes. En las dos versiones realizadas, la participación ha subido desde 67 alumnos en el semestre primavera 2003 hasta 117 estudiantes en el semestre primavera 2005. Los alumnos pertenecen a carreras vinculadas a las áreas de Administración de Empresas y Ciencias Políticas, lo cual ofrece una amplia heterogeneidad, tanto entre estudiantes como entre profesores, generando una discusión de carácter multicultural e interdisciplinaria.

Tabla 1: instituciones y alumnos participantes en la Cátedra Globalización, entre los semestres primavera 2003 y 2005

\begin{tabular}{lcc}
\hline Instituciones & \multicolumn{2}{c}{ Número de alumnos } \\
\hline St. Thomas University, New Brunswick, Canada & 2003 & 2005 \\
The University of St. Thomas, Houston, United States & 13 & 27 \\
Universitat Abat Oliba CEU, Barcelona, España & 21 & 19 \\
Universidad FASTA, Mar del Plata, Argentina & - & 37 \\
Universidad Santo Tomás, Santiago de Chile & 14 & 10 \\
\hline Total alumnos & 13 & 21 \\
\hline
\end{tabular}

Fuente: Estadísticas internas red IC-USTA.

En términos generales, el contenido del curso contempla el estudio de los orígenes de la globalización y el análisis de las principales características políticas, económicas y sociales de este proceso. Estos tópicos se plantean y debaten entre alumnos y profesores localizados en países distintos, exponiendo a los estudiantes a diferentes puntos de vista sobre las causas, desafíos y resultados del proceso de globalización.

Los primeros módulos se destinan a explicar el fenómeno, los países que podrán ser beneficiados y perjudicados por éste y el rol que han desempeñado los principales organismos multilaterales, 
como el Banco Mundial y Fondo Monetario Internacional. Para concluir, estos módulos proponen un nuevo orden financiero como base para una mejor cooperación mundial.

Los módulos siguientes estudian diversas iniciativas regionales de integración como son el North American Free Trade Agreement (NAFTA), el Área de Libre Comercio de las Américas (ALCA), el Asia-Pacific Economic Cooperation (APEC), el Mercado Común del Sur (MERCOSUR) y la Unión Europea, analizadas desde el punto de vista político, económico y social. Se revisan sus principales características, dificultades de implementación y logros; se discuten las ventajas y desventajas de llevar a cabo iniciativas regionales y cómo estas pueden afectar el éxito de un proceso global de integración.

El curso tiene una duración de 15 semanas, entre agosto y diciembre de cada año, con un programa común para todas las instituciones participantes; la evaluación la realiza cada profesor a su respectivo grupo de estudiantes, por separado, y pone énfasis en la participación del alumno en la discusión de los diferentes módulos.

En la Universidad Santo Tomás de Chile la cátedra se ofrece como asignatura electiva a alumnos de la carrera de Ingeniería Comercial.

Los recursos tecnológicos son fundamentales en el éxito de este tipo de iniciativas, debido a que se requiere una plataforma única a la que puedan acceder los académicos y alumnos de las distintas universidades participantes para llevar a cabo la discusión, para generar estadística que permita evaluar la participación en la discusión y para proporcionar acceso a la traducción de los comentarios de cada participante.

Los resultados de las encuestas de evaluación indican que los aspectos más valorados por los estudiantes son: conocer las 
opiniones de alumnos y profesores residentes de diversas regiones del mundo respecto del proceso de globalización, y haber obtenido una respuesta más detallada a sus interrogantes que la que acostumbran a recibir en un clase presencial.

\section{Evaluación de los gobiernos corporativos de las empresas}

La disponibilidad de información sobre los gobiernos corporativos es fundamental para las empresas de un país que desean operar en un contexto global. Este tipo de datos es evaluado cuidadosamente por los inversionistas al momento de tomar sus decisiones de inversión.

Prácticas apropiadas de gobierno corporativo facilitan y, por consiguiente, reducen el costo de levantar capitales en los mercados internacionales para las empresas. Información particularmente relevante en presencia de los escándalos financieros y contables que sembraron la incertidumbre en los mercados durante los primeros años de esta década.

En vista de lo anterior, y con el objetivo de entregar a la comunidad de negocios un instrumento que permitiera la evaluación de los gobiernos corporativos de las empresas del país, se generaron, a comienzos de la década, estándares de gobiernos corporativos en conjunto entre las universidades Santo Tomás de Santiago de Chile y de Houston.

A partir de ese estudio, las empresas del país -e inversionistas nacionales e internacionales- cuentan hoy con estándares de evaluación de los gobiernos corporativos similares a los utilizados en países con mercados de capitales desarrollados, pero adaptados a las prácticas de negocios y realidad legal del país. Fue elaborado por académicos del Centro de Ética y Negocios de ambas instituciones, y dado a conocer a las entidades reguladoras y legislativas chilenas, así como también a investigadores, empresarios 
y medios de comunicación, con el fin de contribuir a la formación de un adecuado gobierno corporativo en el país.

\section{Recursos educativos}

En el área académica, junto al creciente intercambio de alumnos y profesores y a las asesorías interuniversidades, han sido clave los traspasos de información y de programas educativos. La Universidad Santo Tomás de Chile ha sido protagonista de dos experiencias concretas: la primera es la incorporación de nuestra metodología de enseñanza Syllabus en algunos cursos de la UST de Houston; la segunda, la implementación de un centro de aprendizaje creado en Houston, y desarrollado en Chile desde 2001, donde los alumnos acceden a un sistema de tutorías en las áreas de lectoescritura y expresión oral, matemáticas, ciencias y técnicas de estudio.

Por el impacto que han tenido estas iniciativas, la UST fue seleccionada, entre las instituciones de educación chilenas, para exponer el método de enseñanza Syllabus y la aplicación del Centro de Aprendizaje en el último encuentro de universidades de Universia, en España, presentación que fue elaborada en conjunto por las universidades Santo Tomás de Chile y Houston.

Asimismo, desde la creación de la red IC-USTA existe un intercambio fluido de recursos bibliográficos, tanto de títulos como de las revistas académicas editadas por las propias universidades.

\section{Extensión}

La globalización y su impacto en todas las áreas de la educación ha sido uno de los principales temas de las conferencias realizadas en la Universidad Santo Tomás en los últimos cuatro años ${ }^{\mathrm{iii}}$.

iii Todas ellas han sido publicadas como documentos, disponibles en la Universidad Santo Tomás. 
Junto a las cátedras, en las universidades de la red se realizan continuamente conferencias sobre temas de actualidad nacional e internacional, con la participación de académicos de instituciones de IC-USTA, y se publican artículos, investigaciones y ponencias de los académicos en las revistas e informes de las universidades asociadas.

\section{Conclusiones}

Este artículo ha puesto énfasis en la importancia de la globalización en el trabajo presente y futuro de las instituciones de educación superior, y en lo decisivo que resulta avanzar en proyectos innovadores que contribuyan a mejorar la calidad del servicio educacional brindado a los alumnos, teniendo en cuenta que, cada vez más, su desempeño profesional tiene que ver con problemas del mundo global.

Los ejemplos exhibidos destacan la relevancia y potencialidad que reviste formar parte de una red de universidades. La experiencia adquirida a través del curso en línea permitirá, en el futuro inmediato, aumentar convenios similares a los existentes y fortalecer la oferta de cursos a los alumnos y ex alumnos de la universidad, y al público en general.

Asimismo, las restantes experiencias descritas -académicas y de extensión- permiten enriquecer la experiencia educacional y fortalecer lazos con la sociedad y comunidad de negocios del país. 


\begin{tabular}{|c|c|}
\hline \multicolumn{2}{|c|}{ UNIVERSIDADES MIEMBROS OFICIALES DE IC-USTA } \\
\hline ANGOLA & Universidad Católica de Angola, Luanda \\
\hline ARGENTINA & $\begin{array}{l}\text { Pontificia Universidad Católica de Argentina, Buenos Aires } \\
\text { Universidad FASTA de Mar del Plata y Bariloche } \\
\text { Universidad del Norte Santo Tomás de Aquino, Tucumán }\end{array}$ \\
\hline AUSTRALIA & Australian Catholic University, Sydney \\
\hline CANADÁ & Saint Thomas University, New Brunswick \\
\hline COLOMBIA & Universidad Santo Tomás, Bogotá \\
\hline CHILE & Universidad Santo Tomás \\
\hline ESPAÑA & $\begin{array}{l}\text { Universidad San Pablo CEU, Madrid } \\
\text { Universidad Cardenal Herrera CEU, Valencia } \\
\text { Universitat Abat Oliba CEU, Barcelona }\end{array}$ \\
\hline ESTADOS UNIDOS & $\begin{array}{l}\text { University of Saint Thomas, Houston, Texas } \\
\text { Aquinas College, California }\end{array}$ \\
\hline FILIPINAS & $\begin{array}{l}\text { University of Santo Tomas, Manila } \\
\text { Aquinas University, Legazpi }\end{array}$ \\
\hline FRANCIA & $\begin{array}{l}\text { Institut Catholique d'Études Superieures, } \\
\text { La Roche Sur-Yon }\end{array}$ \\
\hline INDONESIA & Universitas Katolic Santo Thomas, Sumatra \\
\hline IRLANDA & Mary Inmaculate College, Limerick \\
\hline ITALIA & Pontificia Università San Tommaso d'Aquino, Roma \\
\hline MOZAMBIQUE & Universidad Santo Tomás, Maputo \\
\hline NIGERIA & Catholic University of Nigeria, Abuja \\
\hline PERÚ & Pontificia Universidad Católica de Perú, Lima \\
\hline REPÚBLICA DOMINICANA & Pontificia Universidad Católica Madre y Maestra, Santiago \\
\hline
\end{tabular}

Recibido: 26 de mayo de 2006

Aceptado: 10 de junio de 2006 